\title{
The Present Position of the Wave Theory of Light.
} By Dr. R. A. Houstoun.

I.

THE emission theory of light prevailed for a century after Newton's death. During this time his "Opticks" was regarded as of equal importance as his "Principia," and his emission theory as of equal value as his law of gravitation. Then, principally owing to the work of Fresnel, the emission theory was overthrown, and the wave theory established in its place. The latter in its turn has prevailed for a century, but now in certain quarters doubts are being expressed as to whether it is competent to explain the results of recent experimental work, and whether, after all, it may not be advisable to hark back to some form of emission theory, at least for certain fields of work.

There are two great differences between the situation now and as it existed a hundred years ago. Then the wave theory under Fresnel presented a clear and definite alternative to the emission theory of Newton, explaining certain decisive experiments in a simple and natural manner. The critics of the wave theory at present are not so much hostile as neutral towards it. They present no alternative to it; they admit its strong position and also admit the impossibility of Newton's emission theory in the light of the experimental work of to-day. But they direct attention to certain results which they have difficulty in reconciling with the wave theory, and hint at somehow combining the advantages of both theories.

Another difference between now and a hundred years ago is the manner in which we regard our theories. Then a theory was true or false; we were engaged in interpreting the processes of Nature which existed independently of us and outside of us, and it was necessary that the true solution should be true for all time. Nowadays we do not so much speak of the truth of a theory as of its utility, or rather the truth of a theory lies in its utility. Truth is what works. Consequently we require of a theory only that it should be true for our day and our generation. A theory works if it connects the facts together and enables us to predict new facts. We can never penetrate to the essential nature of things ; we can only compare them with other things. Physical theories are metaphors. When we say that light is propagated in wave motion, we mean that it is propagated like wave motion. This change in the attitude of the physicist towards his theories had been pretty widely adopted before the results of the principle of relativity became known; the latter made the change of attitude known to the public.

The criticisms directed against the wave theory at present arise from two quarters, namely, the principle of relativity and the quantum phenomena.

The special theory of relativity requires that the mass of a system should vary with its interna! energy, and that consequently radiation, including light waves, should have mass.

No. 2705 , VOL. 108]

In connection with this result a paper by Sir Joseph Thomson, entitled "Mass, Energy, and Radiation," which appeared in the Philosophical Magazine for June, 1920, is of the greatest interest and importance, not so much for its actual results as for the development it foreshadows. It is well known that the relativists operate with symbols and not with physical ideas; this paper is " an attempt to help those who like to supplement a purely analytical treatment of physical problems by one which enables them to visualise physical processes as the working of a model; who like, in short, to reason by means of images as well as by symbols."

The paper assumes that all mass, that of atoms as well as that of electrons, is distributed through space with a density determined by the electric field at the place where the mass is supposed to exist, and that energy of every kind, kinetic, potential, thermal, chemical, or radiant, is of one and the same type, being the kinetic energy possessed by the particles which are supposed to constitute mass. Transformation of energy is merely the flow of the mass particles from one place to another. Thus, for example, on this view, when a body gains kinetic energy, it is not because any of its mass particles are moving faster; it is because the mass of the body has been increased, and the increase in the mass implies a proportional increase in the energy.

We are not yet in a position to calculate the mass of any one of these mass particles, but at least ${ }_{10}{ }^{11}$ are required to supply the mass of one electron. If energy is indivisible beyond a certain limit, the inverse square law of electrical attraction cannot hold over more than a certain finite distance.

The distribution of these particles and their movement from one place to another are determined by the distribution of the lines of electric force. In addition to mass particles it is assumed that there are in the universe lines of force spreading through space, the electron being at one end of a line of force and a unit of positive electricity at the other. The mass particles are concentrated in the places where the electric field is strongest. Thus, for example, if the radius of an electron is Io ${ }^{-13} \mathrm{~cm}$., onlv one-thousandth part of its mass will be at a distance from the electron greater than $10^{-10} \mathrm{~cm}$. The mass particles perform the functions of both æther and matter. Comparing the physical universe with a living organism, we may regard the mass particles as the flesh and the lines of force as the nervous system.

A light ray is consequently a jet of particles and lines of force moving sideways, the density of both varying periodically along the jet. Refraction is explained by the action of the secondary waves emitted by the electrons of the refracting medium under the stimulus of the incident wave.

The paper is noteworthy, because it points the @1921 Nature Publishing Group 
way we shall have to travel if the new ideas are to be translated into everyday physics in all their original force. It also prompts the query whether it is worth while.

The special theory of relativity disturbed the generally accepted views about the æther by giving equal value to co-ordinate systems moving with uniform velocity with reference to one another. We had always thought of an æther at rest, through which the sun and the planets moved, and in which our ultimate system of co-ordinate axes was at rest. The most straightforward interpretation of the special theory of relativity is to give each planet, each moving electrical charge, its own æther, and at the same time to remove all substantiality from the very great number of æthers thus postulated. The plain man wants to know, if the light comes from the sun, in which æther it travels, the sun's æther or the earth's, and if there can be wave motion without a medium. This question of the æther has been discussed so fully, and there are so many different views, that it will be passed by here with the suggestion that possibly the special theory of relativity makes too great demands when it asserts that all moving systems have equal value. The system that the inhabitants of the earth are moving with possesses a. special value for them and their physical theories, because they are moving with it. We only ask of Boyle's law, for example, that it should hold for the temperatures that we can produce in the laboratory, not for impossibly high temperatures that we can never attain. In the same way it is asking too much of the wave theory of light in the form we use it that it should be equally useful (and true) for us and the possible inhabitants of Mars. It is dangerous to attribute universal validity to theories which can be tested only in a limited class of cases. Consequently the æther moving with the earth is the æther. Again, with reference to the apparent unsubstantiality conferred on the æether by the principle of relativity, it is forgotten that it confers some unsubstantiality on everything else as well, even the water that water waves travel in.

The general theory of relativity required that light should be deflected on passing close to the sun's surface, and, as is well known, this deflection has been verified experimentally by the observations made by the rgig solar eclipse expeditions. On the relativity theory the space in the sun's gravitational field is non-Euclidean, and the deflection is caused simply by the properties of space. The fact of the deflection is so much simpler than the explanation that it seems probable that the physicist will ignore the latter. One wonders if it is possible to treat the deflection geometrically in a simpler manner directly from the postulate of parallels. There has been an unsuccessful attempt to explain the deflection by an emanation of matter from the sun and a consequent increase of refractive index in its neighbourhood. Newton's emission theory gives a deflection of exactly half the required amount; so also does the electro- magnetic theory, if we make the unusual assumption that ordinary mass is associated with the energy of the wave, and that this mass is acted on by gravity. At present there seems no satisfactory alternative to a non-Euclidean geometrical optics and wave theory, but it is probably better to wait and in the meantime to suspend judgment.

The existence of the quantum was discovered theoretically in Planck's celebrated theory of radiation. It will be advantageous to give an account of this theory here, because an important modification of it has strengthened the view that there is nothing in the quantum phenomena inconsistent with classical mechanics or electrodynamics. This modification came too late to be noticed in certain widely read descriptions of the theory published in this country, and it has consequently received little attention.

If a hollow vessel is maintained at a uniform temperature, and radiation allowed to issue from a small hole in its side, the intensity of the radiation and the spectral distribution of its energy are independent of the material of which the vessel is made. The rays are reflected forwards and backwards inside the vessel before they issue, and any initial difference in intensity is evened out by the successive reflection. In order to derive a theoretical value for the spectral distribution of the radiation issuing from such an enclosure at different temperatures_- "black" radiation, as it is called-Planck assumed that there were in the enclosure a great number of oscillators or vibrators, small Hertzian doublets, all of the same frequency, and in a state of equilibrium, radiating and absorbing energy. The total energy of the system remained constant, but the energy of the different oscillators was not the same; there were always some gaining and some losing energy. Moreover, this exchange took place solely by scattered radiation; there was nothing in the nature of corpuscular radiation or characteristic radiation taking place. The distribution of the energy among the different oscillators occurs according to the laws of probability, and by using a general definition of temperature the temperature of the system can be derived from this distribution of energy. Then the density of the radiation in the enclosure can be calculated for the particular frequency in question. In order to obtain the correct value, namely :

$$
\mathrm{E}_{\lambda}=\frac{c_{1}}{\lambda^{5}} \frac{\mathrm{I}}{e^{c_{2} / \lambda \tau}-\mathrm{I}}
$$

it was necessary to assume that the emission of energy took place discontinuously in whole multiples of the quantum, the quantum being defined by $\epsilon=h v$, where $v$ is the frequency of the radiation and $h$ a universal constant, Planck's constant. This emission of radiation in quanta was opposed to all previous ideas.

The criticism which the experimental physicist naturally passes on Planck's proof as outlined above, and as described in his "Vorlesungen über die Theorie der Wärmestrahlung" (second edi-

$$
\text { No. } 2705 \text {, VOL. IO8] }
$$


tion, $191^{3}$ ), is that in practice the energy changes do not take place by scattered radiation alone, but also by corpuscular radiation and characteristic or fluorescent radiation. It does not seem permissible to consider scattered radiation by itself. The genesis of radiation must involve the mutual play of both corpuscular radiation and waves. When $\mathrm{X}$-rays fall on a body some of the incident energy reappears as scattered radiation, some as corpuscular radiation, and some as characteristic radiation. Consequently Planck's original oscillators formed an artificial body which has no counterpart in reality. He was, of course, aware of this, for on p. I 33 he states that "it does not matter whether such a body exists anywhere in Nature, it is only necessary that its existence and properties should be compatible with the laws of electrodynamics and thermodynamics."

As a result of the difficulties associated with the form of the theory described in the book referred to above Planck made an important modification of his hypothesis (" Eine veränderte Formulierung der Quantenhypothese," Preuss. Akad. Wiss, Berlin, Ber. 34, pp. 918-23, I9r4). This paper assumes that radiation and absorption take place continuously, and that the quantum action is not between the oscillators and the radiation, but takes place between the oscillators and the free particles (molecules, ions, and electrons), which exchange energy by impacts with the oscillators. The laws of classical electrodynamics then hold good for every interchange between the oscillators and free radiation. At the same time the radiating substance becomes more like its counterpart in Nature, and the feeling of artificiality which the former theory produced is removed. Also the difficulty connected with the use of Hertz's expression for calculating the density of the radiation disappears.

(To be continued.)

\section{The Extent of the Recent Drought.}

THE recent prolonged drought in the British Isles has directed attention to an interesting aspect of meteorological science. It is natural to inquire how far the drought has been confined to our immediate neighbourhood, or how far it has been general. With the exception of Hildebrandsson's pioneer work on action centres, no systematic research dealing with the extent to which drought has affected considerable areas of the earth's surface at one time has yet been carried out. A basis for detailed study of this character will be provided by the "Réseau Mondial," published by the Meteorological Office, five annual volumes of which have now been issued. This publication gives pressure, temperature, and rainfall for about 400 stations distributed over the globe, the month being taken as a unit. In the present article it is proposed to make a preliminary survey, so far as material is already available, of the world's weather this year, particularly during the months May, June, and July. As no system of telegraphic reporting from "Réseau Mondial" stations has yet been established, we have to rely in making such a survey on the most recent monthly, weekly, or daily weather reports obtainable from the various countries, and, largely, upon general newspaper reports.

Table I. shows the percentage of normal rainfall which has fallen in various parts of the British Isles since the beginning of the year:-

\section{TABLE I.-Percentage of Normal Rainfall.}

\begin{tabular}{|c|c|c|c|c|}
\hline $\begin{array}{c}\text { rga2r. } \\
\text { January }\end{array}$ & $\begin{array}{l}\text { England and } \\
\text { Wales. } \\
\text { I46 }\end{array}$ & $\begin{array}{l}\text { Scotland. } \\
\text { I } 68\end{array}$ & $\begin{array}{l}\text { Ire'and. } \\
\text { II } 9\end{array}$ & $\begin{array}{c}\text { British Isles. } \\
\quad x_{45}\end{array}$ \\
\hline February & 15 & 39 & 51 & 34 \\
\hline March ... & Ior & 170 & X 29 & 133 \\
\hline April ... & 59 & 61 & 46 & 56 \\
\hline May $\ldots$ & 79 & 108 & 90 & $9 \mathrm{r}$ \\
\hline June $\quad \ldots$ & 17 & 40 & 24 & 26 \\
\hline July & $\begin{array}{l}\text { Probably } \\
\text { below 50 }\end{array}$ & $\begin{array}{l}\text { Rather } \\
\text { above } 100\end{array}$ & A bove 100 & About roo \\
\hline
\end{tabular}

Table II. gives the percentage of normal rainfall for the various districts into which the British Isles are subdivided:-

\section{TABLE II.-Percentage of Normal Rainfall by Districts.}

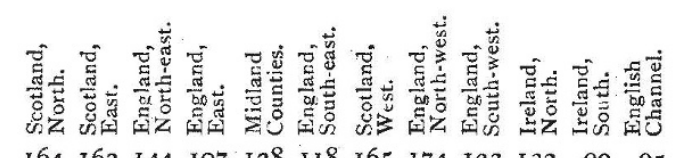

$\begin{array}{llllllllllllll}\text { January } & 164 & 162 & 144 & 107 & 128 & 118 & 165 & 174 & 123 & 132 & 99 & 95\end{array}$ $\begin{array}{lllllllllllll}\text { February } & 64 & 26 & 16 & 26 & 15 & 21 & 37 & 14 & 9 & 38 & 60 & 15\end{array}$ $\begin{array}{lllllllllllll}\text { March... } & 160 & \text { I09 } & 45 & 53 & 73 & 62 & 176 & \text { I24 } & 92 & \text { I27 } & 114 & 67\end{array}$

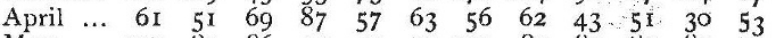
$\begin{array}{llllllllllllll}\text { May } & \ldots & 117 & 89 & 86 & 59 & 70 & 71 & 500 & 83 & 89 & 89 & 83 & 73\end{array}$ $\begin{array}{lllllllllllllll}\text { June } & \cdots & 46 & 4 \mathrm{I} & 27 & 20 & 18 & 6 & 30 & 15 & 13 & \text { I } 7 & \text { 10 } & 36\end{array}$ Average percentage February to June :$\begin{array}{llllllllllll}90 & 63 & 49 & 49 & 47 & 45 & 80 & 60 & 49 & 64 & 59 & 49\end{array}$

It should be noted that Tables I. and II. are not based on identical stations.

Table I. shows that January was a month of excess rainfall in all regions. Previous to this we have to go back to July, 1920, to find another month with rainfall above normal for the whole British Isles, the percentages for August to December, I920, varying between 68 and 96 . It is evident from the table that the drought has been much more conspicuous in England and Wales than in Scotland and Ireland, where it has not been so remarkable. This is well shown in the map (Fig. I), which has been prepared by the British Rainfall Organization. The area of greatest drought is the southern and eastern midlands, the amount of rainfall increasing outwards from this centre, particularly to the north and west. February, April, and. June were the months of greatest deficiency. March, which appears to be normal (IOI per cent.), was a month of drought in most places in the eastern and midland counties, but wet in the west and north-west. 